International Journal of Advanced Trends in Computer Science and Engineering

Available Online at http://www.warse.org/IJATCSE/static/pdf/file/ijatcs240932020.pdf

https://doi.org/10.30534/ijatcse/2020/240932020

\title{
NOC'S: Buffered and Bufferless Structure and their design methodologies for High throughput and Low latency
}

\author{
${ }^{1}$ Sujata. S.B, ${ }^{2}$ Anuradha M Sandi, \\ ${ }^{1}$ Research Scholar, Department of Electronics and Communication, GNDEC Bidar \\ VTU Belgaum, sujatabidreddy@gmail.com \\ ${ }^{2}$ Professor, Department of Electronics and Communication, GNDEC Bidar, anu29975@gmail.com
}

\begin{abstract}
To know and meet the existing issues and demands related to scalability of number of nodes, their sizes of Network on Chip (NoC) which are important networks for efficient communication to transfer multimedia data at low latency and high throughput. These NoC's was designed and developed the chips for both 2D and 3D which includes switching, routing and crossbar techniques, out of which routing algorithms are plays major role due to the computational operations take place here and produce the different delay. In turn these delays are affected on throughput and latency. Many of the NoC's has buffered and bufferless routers to optimize the area, power consumption and latency. Therefore the detailed literature survey has been done mainly on latest buffered and bufferless NoC's with different routing algorithms used for addressing the major constraints like QoS, throughput, latency and hardware utilizations for different injection rates. As per requirements at present situation for better communication within core networks, this paper mainly focused on commonly used architectures and their inter components connectivity's that can deal with methodologies and design challenges for optimization of signal integrity, scalability, throughput and latency in an NoC's. At the last, modified buffered and bufferless NoC architectures are proposed which includes the modified routing algorithm called Dynamically buffered and bufferless reconfigurable NoC ( $\mathrm{DB}^{2} \mathrm{R}-\mathrm{NoC}$ 's) and feasible direction finding (FDF) for switching. Making use of these architectures, any dimensions of NoC's can be designed and can validate for multimedia data and also can test on Field Programmable Gate Array (FPAG) processors.
\end{abstract}

Key words: Buffered, bufferless, ECC, Routing, NoC, QoS, Security system, and FPGA

\section{INTRODUCTION}

In network communications, the data transmissions are major constrains with high throughput and low latency and also delay, area, power consumptions are the important parameters for System on Chips and Network on Chips. On-chip interconnection networks employ buffer-less routing which is one the advantage of these networks. Router input/output buffers are eliminated in buffer-less routing. Hence, substantial energy drops can be achieved at modest performance loss in contrast to buffered routing algorithms. In most real applications, the volume of the introduced traffic is not very high. Since router latency is low, buffer-less routing achieves high performance. We assume that buffer-less routing algorithms can simplify network and router design by reducing multifaceted buffer management/distribution techniques. Thus, this could be the process of selection for interconnection networks that are known to operate the output at below-peak. Buffer-less network strategy has many drawbacks in terms of functionalities which has been overcome by buffered networks such as support for starvation freedom/avoidance, QoS and various traffic service programs, fault tolerance with faulty links/routers, congestion realization, and energy management. Buffer-less routing algorithms incorporates these supports which is the main aim of our future work.

Exact scale multicore systems are utilized in applications such as IoT, cognitive computing, and cloud computing that are based on on-chip networks which is one of the important shared resources. The appropriate usage of it will lead to substantially improved energy efficiency. Due to the chip area and power consumption constraint, the NoC has drawbacks associated with energy efficiency and average performance. Common multicore loads are recommended for a mono-NoC while increasing the prerequisites for resources and application features, the system performance is confined by severe interference between applications. To set up a mono-NoC for huge data stacks, enhancing the quality of service and energy efficiency under massively integrated stacking of applications is done by employing hybrid NoCs which is based on the application-aware design. This paper initially proposed a hybrid NoC with a dedicated bufferless $\mathrm{NoC}$ and a buffered NoC, as well as an application-aware mechanism supporting in choosing optimal efficient NoC. We proposed a novel metric NC_ratio to assess the huge data stack. We analyzed both 64-thread and 128-thread systems, and our proposed method demonstrates the important developments in system performance. 
Furthermore, we proposed a novel congestion optimization algorithm for hybrid NoCs. It is carried out by monitoring the congestion status of various NoCs and reallocating the packets in the congesting nodes. The simulation results of the proposed two methods illustrate that the energy efficiency of the complete system can be considerably increased.

\section{LITERATURE SURVEY}

To describe the problems of area and power in the on-chip network, in this paper, we have recommended an area-benefit method MaS for buffer-less on-chip network, which facilitates the following transfer without large buffering requirements at the receiver end and maintaining energy efficiency. In contrast, with recently proposed deflecting bufferless routing BLESS-Worm, MaS decreases the rising buffering requirements at the receiver end which is induced by the faulty reports. Furthermore, the deadlock and livelock freedom of the MaS algorithm has been demonstrated [1]. Correct simulations of the Extensive cycle have been executed to illustrate that $\mathrm{MaS}$ provides better performance in contrast to BLESS-Worm. Results depict that, MaS reduces average packet latency by $10 \%$ and power consumption drops by $9 \%$, respectively. Additionally, at the receiver end, the buffer requirements are reduced up to $80 \%$. For future design, the study of real traffic will be done. Besides, the routing algorithm should be estimated in other significant ranking policies such as the most deflecting-first method [3].

Based on the design of high performance and efficient routers, the efficiency and performance of an NoC are dependent. In this thesis, we proposed ReDC, a bufferless deflection router that employs two PDN with identical inputs set, but specified in a different order, to obtain maximum productive output ports. The proposed scheme provides better performance by choosing the PDN which presents a greater number of productive output ports so that the deflection rate of the flits is reduced and the average flit latency is negligible for the similar critical path latency when compared to the advanced bufferless deflection router [4].

In [2], various periodic scheduling algorithms for bufferless NoCs are presented, that are designed to overcome the complex communication parameters of real-time applications, applications associated with the BSP programming model, and network congestion-control applications. In particular, we introduced DTNS and TNS scheduling algorithms that were proved to be optimal for complete exchange traffic on degree-two and torus networks. Specifically, we present DTNS and TNS scheduling algorithms that were verified to be optimal for complete exchange traffic on degree-two and round-shaped networks. Application of the TNS algorithm is implemented on mesh NoCs, and it accomplishes a constant bounded schedule length in contrast to the optimal scheduling [2]. The complete-exchange traffic pattern presents an assured structure that can also be employed for other patterns, which requires constant communication between the nodes, keeping some of the programming slots empty. Later, a general periodic traffic pattern is provided by the Latency-based scheduling algorithm. We illustrate that the latency-based scheduling algorithm is more efficient than random materialistic scheduling on NoC topologies such as rings, tori, and mesh. In future work, we insist to study the following public issues: demonstrating the drawback of collision-free scheduling for periodic arbitrary traffic is NP-hard, developing our Latency-based algorithm, and to apply our results to other collision-free networks, such as optical networks, which comprises of arbitrary topologies [5].

In [6], propose BLOCON, silicon photonics is employed to develop a bufferless Clos network architecture. A $64 \ldots 64$ BLOCON decreases the thermal tuning power of the PXBar from $2.6 \mathrm{~W}$ to $0.39 \mathrm{~W}$ and has a $100 \%$ bisection bandwidth under uniform traffic. Wormhole routing is employed by BLOCON and is regarded as an input queued switch without reassembly queues or $\mathrm{VCs}$ in the output port. Lack of multi-stage routers allows BLOCON to experience high output with an appropriate scheduling algorithm. In this paper, two scheduling algorithms have been proposed Sustained and Informed Dual Round-Robinn Matching (SIDRRM) and Distributed and Informed Path Allocation (DIPA) to resolve the output argument and Clos network routing issue. We also proposed an approach to enhance the off-chip laser-power consumption by evaluating the optimum number of wavelengths multiplexed in the waveguides and by fine-tuning the position of SMs according to the long-term average traffic load. The simulation results illustrate that both SIDRRM and DIPA outrun the existing scheduling algorithms on BLOCON. The simulation results also demonstrate that BLOCON with SIDRRM and DIPA exceeds the compared photonic and electrical NoC architectures in terms of 100-cycle throughput and on-chip power efficiency under synthetic traffic patterns and SPLASH-2 traces [6].

In [7], a fault-tolerant solution for a bufferless NoC is presented to secure it from both transient and permanent faults on the links. A detailed chapter of this paper can be defined as follows.

1) SECDED Hamming code is used by on-line fault diagnosis mechanism to identify both transient and permanent faults, based on the distribution of the faults over the link, the encoding scheme can correct between 1-7 faulty bits and detect between 2-14 faulty bits.

2) A hybrid ARQ/FEC scheme, which can accomplish symmetrical break down even at a high fault rate, is proposed to endure transient errors during transmission.

3) The FTDR algorithm, which assures the "0 lost packets" reconfigures the routing table via advanced learning technique to route packets by preventing permanent faults.

To reduce the costs of the router area, a hierarchical-routing-table-based algorithm (FTDR-H) is conferred. The FTDR and FTDR-H routers exceed the other 
Sujata. S.B et al ., International Journal of Advanced Trends in Computer Science and Engineering, 9(3), May - June 2020,4117 - 4127

two fault-tolerant deflection routers. The practical results exhibited that FTDR and FTDR-H routers are high-reliability bufferless routers, which can protect against any fault distribution pattern, as long as the network is not divided into two or more disconnected sub-networks. For miniature networks (e.g., less than 64 nodes) the FTDR router is cost-efficient, whereas the FTDR-H router has good scalability and provides an accurate solution for at least several hundreds of nodes. The significant drawback of this paper depends on the following features: 1) a faulty link has to be stopped bidirectionally and 2) for only one cycle transient faults are concluded. In future work, we will portray these issues and analyze more complex fault models, which will exhibit real fault circumstances in CMOS technologies of 20 $\mathrm{nm}$ and below [7].

With the declining technology sizes and rising cores number which are integrated on a single chip, the implementation of fault-tolerant NoCs is crucial when its performance reduces. In this paper, we recommend QORE - a fault-tolerant NoC architecture that utilizes reversible channel buffers. We employ QORE's reversibility for enhanced performance and to get-rid of faulty links. We also develop structures and employ decision hierarchies to forecast traffic direction on the links to recover the link controllers. Empirical results illustrate that a decision tree forecasts the direction of the traffic with higher precision on average than a predictor based on the application of the threshold link. Our results on actual standards (SPEC CPU2006, PARSEC, and SPLASH-2) portrays an increase in acceleration of $1.3_{-}$and enhanced overall output by $2.3_{-}$on artificial traffic associated with related work. By employing Synopsys design compiler, we demonstrate that QORE decreases network power by $21 \%$ while lacking minimal control overhead [8]. The bestowed RoShaQ, a new router architecture that permits sharing multiple buffer queues for increasing the output of the network. The shared queues are avoided by the input packets to attain low latency where the network load is less. In contrast to synthetic traffic patterns, the standard VC router as an identical buffer area also has $17 \%$ lower zero-load latency and $18 \%$ higher saturation throughput on average with only $4 \%$ higher power and $16 \%$ larger area. It also consumes $5 \%$ higher throughput than a full-crossbar VC router with 3\% lower power and 3\% less area. All the routers start saturating at an equivalent average packet latency of 100 cycles, RoShaQ had 9\% and 7\% lower energy dissipated per packet than standard VC and full-crossbar VC routers, respectively. We also introduced a technique for evaluating and comparing the performance and energy-efficiency of routers over real multitask embedded applications. Over these applications with random mapping, RoShaQ had 26\% and $12 \%$ lower latency than standard VC and full-crossbar VC routers, respectively, while aiming the same inter-task communication bandwidth requirements. In terms of energy, RoShaQ consumes $23 \%$ and $14 \%$ lower energy per packet than conventional VC and full crossbar VC routers, respectively. By employing the NMAP mapping algorithm,
RoShaQ realized higher development in application performance and energy consumption than other routers. On average, RoShaQ had $32 \%$ and $28 \%$ low application latency and consumed $30 \%$ and $33 \%$ less energy per packet than VC and full-crossbar VC routers, respectively [9].

We have illustrated that to reduce the performance costs and increase the NoC implementation, the buffering size of each input channel has to be prudently assigned to match the characteristics of application traffic. An efficient desirous algorithm was proposed, which automatically distributes the buffering resources to various NoC channels, such that the communication performance is enhanced with an adequate total buffering resource budget. The selection of a 2-D mesh network as the fundamental NoC architecture serves typically as an example. Our algorithm can be drawn-out to arbitrary topologies by modifying the analytical models correspondingly to the target topology. Additionally, we have designed our algorithm only for NoCs with $X Y$, OE-fixed, and OE-split routing, the method is typical and can be applied to other deterministic and unconscious routing schemes meanwhile, in these instances, the arrival rate of each channel can be computed. We proposed to develop this research in several directions. Extending this technique to NoCs that support adaptive routing is of the one probable direction. The most important confront comes from the complexity involved in the computation of the arrival rate for each channel as in adaptive routing multiple routing paths are possible. One more significant extension is to adapt NoCs with wormhole switching. Lastly, we employed a field-programmable gate array archetype and designed to utilize it for the exact estimation of the effectiveness of the buffer allocation algorithm [10].

The 3-D network-on-chip (NoC) router is one of the most important causes of thermal hotspots, which reduces the performance gain of 3-D integration. Due to the variable cooling efficiency of various silicon layers in 3-D NoC, the optimal conditions of a conventional load balancing design (LBD) scheme and temperature balancing design (TBD) scheme may not be satisfied. To evaluate the tradeoff between performance and temperature, we present a novel analytical model. The model demonstrates that the LBD scheme and the TBD scheme can be contemplated as two corner instances in the design area, and design instances can be characterized by comparing the bandwidth bound and the thermal-limited bound. To detect the optimal design conditions between the LBD and the TBD schemes in 3-D NoC, we develop a novel routing-based traffic migration, vertical-downward lateral-adaptive proactive routing (VDLAPR), and buffer allocation techniques, vertical buffer allocation (VBA). The VDLAPR algorithm allows a tradeoff between the LBD and the TBD schemes. The proposed VBA technique reduces traffic congestion induced by traffic migration. To achieve optimum configuration, we recommend a systematic design flow, which helps in determining the best design parameters in the extended area between LBD and TBD. Depending on 
Sujata. S.B et al ., International Journal of Advanced Trends in Computer Science and Engineering, 9(3), May - June 2020,4117 - 4127

the traffic-thermal co-simulation analysis, the feasible output can be increased from $2.7 \%$ to $45.2 \%$ by employing the proposed design system [11].

The significant design measures of networks-on-chip (NoCs) are end-to-end delay, output, energy consumption, and silicon area. However, initially, various analytical models have been proposed for forecasting such measures in NoCs, only limited of them reflect the effect of message delaying time in the buffers of network routers for predicting overall power consumption and none of them contemplate structural heterogeneity of network routers. This paper presents two inter-related analytical models to evaluate message latency and power consumption of NoCs with arbitrary topology, buffering structure and routing algorithm. Buffer distribution scheme describes the buffering area for each channel of the $\mathrm{NoC}$ that can either be homogenous (all channels having similar buffer structures) or heterogeneous (each channel having its buffer structure). Therefore, the buffer allocation scheme can be either homogenous or heterogeneous. We infer that for a physical channel, bandwidth sharing of virtual channels is not possible, and by employing Poisson distribution IP cores generate messages. From simulation results, we substantiate that the proposed models show adequate accuracy for various network configurations working under different operating conditions. We have presented the analysis on a Poisson traffic model which is beneficial and is utilized for scenarios with real application workloads [12].

Conventional input-queued routers in network-on-chips (NoCs) merely have a few numbers of virtual channels (VCs) and packets in a VC are defines in a fixed order. Such a specific design is responsive to head-of-line (HoL) blocking only the packet at the head of a $\mathrm{VC}$ and can be assigned by the switch allocator. In this paper, reorder buffer (RoB) techniques are proposed which is used to schedule packets in input buffers. Later in on-chip routers, switch allocation is an evaluative pipeline stage, the performance of NoCs are drastically reduced by HoL blocking. We design VCs as RoBs to allow packets to be positioned before the head packets and not at the head of a VC. The arguments in switch allocation are decreased by RoBs and also reduces the HoL blocking and thus enhance the NoC performance. On the other hand, due to circuit complexity and power overhead, it is difficult to rearrange all the components in a VC. We design RoB-Router, which controls variable RoBs in VCs and permits only a portion of a VC to act as RoB. Depending on the number of buffered flits RoB-Router automatically decides the length of RoB in a VC. This scheme achieves excellent efficiency by reducing the resource. Also, to enhance the performance of RoB-Router we propose two independent approaches. One approach is to restructure the VC allocation strategy by improving the packet order in input buffers. The RoB-Router is combined with an existing efficient switch allocator TS-Router is another approach. The evaluated results illustrate that our design can accomplish
$46 \%$ and $15.7 \%$ performance enhancement in packet latency under synthetic traffic and traces from PARSEC than TS-Router, and the cost of energy and area is reasonable. Furthermore, average packet latency is decreased by our two successful approaches and uniform traffic is $13 \%$ and $17 \%$ respectively [13].

The performance of Networks-on-Chip can be significantly increased by Multicast communication. At present most of the multicast routing algorithms are either tree-based or path-based. The previous scheme had low latency but it is necessary to solve the problem of multicast deadlocks through additional hardware resources. Later we can evade deadlocks easily but long routing paths are required for it. In this paper, we design a hybrid multicast routing method that integrates the advantages of both paths- and tree-based methods. The proposed scheme guarantees deadlock-free multicast routing without involving additional virtual channels or large buffers to hold large packets. By employing an adaptive routing strategy and taking into account the traffic load in nearby routers we can achieve high routing performance. Two approaches, particularly node balancing and path balancing, are further established to develop this hybrid routing algorithm. Many experiments with various buffer sizes, packet sizes and numbers of destinations per packet under random and NoC rule traffic at different traffic injection rates have been shown. The results depict that the average latency of our method is lesser than prior multicast routing algorithms in most instances, and the saturation points of our method are constantly significant at higher injection rates [14].

Router's buffer design and management extremely impact on energy, area, and implementation of on-chip networks; hence it is difficult to include all of these parameters in the design process. Simultaneously, the NoC design cannot ignore avoiding network-level and protocol-level deadlocks by allocating ad-hoc buffer resources to that purpose. In Chip Multiprocessor Systems (CMPs) the coherence protocol typically requires diverse virtual networks (VNETs) to prevent deadlocks. Furthermore, the VNET application is highly unstable and there is no mode to share buffers between them due to the inevitability of separating different traffic categories. This paper suggests CUTBUF, a new NoC router architecture to dynamically allocate VCs to VNETs reliant on the actual VNETs load to considerably decrease the number of physical buffers in routers, therefore saving area and power without reducing NoC performance. Additionally, CUTBUF lets to use the same buffer again for various traffic kinds while guaranteeing that the enhanced NoC is deadlock-free at both network and protocol level. In this viewpoint, all the VCs are contemplated and additional queues are not statically allocated to a precise VNET and the coherence protocol only enforces a minimum number of queues to be performed. Synthetic applications, as well as real standards, have been employed to authenticate CUTBUF, taking into account architectures ranging from 16 up to 48 cores. Likewise, a 
complete RTL router has been proposed to analyze area and power costs. Results show in what way CUTBUF can lower router buffers up to $33 \%$ with $2 \%$ of performance degradation, $5 \%$ of operating frequency reduction and area and power-saving up to $30.6 \%$ and $30.7 \%$, respectively. On the other hand, the flexibility of the suggested architecture enhances by $23.8 \%$ the performance of the baseline NoC router when the identical buffers are employed [15].

This paper presents the perception of $F P D$ and proposes the $P D A-F T R$ algorithm to attain fault-resilient packet delivery and to balance the traffic load. Depending on the FPD information, and to select the path we combine it with local buffer information. However, we relate our work with other associated works in terms of saturation output, fault-tolerance ability, traffic-balancing ability, and performance scalability. The experiments demonstrate that better performance in a faulty $\mathrm{NoC}$ is achieved by a complete PDA-FTR. The proposed router architecture substantiates that our suggested design is cost-efficient [16]. The requirement for advanced output and lower communication latency in contemporary networks-on-chip (NoC) has escorted to low- and high-radix topologies that utilize the speed provided by on-chip wires-after proper wire engineering- to transmit flits over longer distances in a single clock cycle. In this paper, we are provoked by the fast link traversal principle, we design the Rapid Link NoC architecture, which achieves the speed quickly to transfer flits between end-to-end routers using double-data-rate (DDR) link traversals. Rapid Link is improved with new low-cost DDR flexible buffers that pipeline link traversal (when required) to multiple flow-controlled half-cycle segments, whereby both the positive and negative edges of the clock are driven by each portion of data. DDR link traversal leads to multiple NoC configurations that can significantly increase network performance without enhancing the area/power cost of the NoC comparative to advanced single-data rate architectures. Cycle-accurate network simulations are wide in number, the efficiency of Rapid Link and its potential to scale NoC architecture [17] is illustrated by the results obtained after hardware execution.

In [18], we have designed a flit-level accelerating scheme to increase the NoC performance by utilizing self-reconfigurable bidirectional links. To assist the transferring of two flits in the same packet at the same clock cycle, to determine the link directions dynamically at run time, a novel link direction managing scheme has been proposed. The input buffers and the switch allocators are also modified to permit two flits from the identical packet to take part in the data transmission. Similarly, we have extended the CDC structure to operate under the contemporary long wire links. The operating hardware cost is also studied in this paper. From synthetic and real application traffic simulation results, it is depicted that FS NoC enhances the latency and throughput performance with reasonable operating costs [18].
One of the evolving nonvolatile memory (NVM) technologies is Spin-Torque Transfer Magnetic RAM (STT-RAM) which is considered as the spare for SRAM memory architectures and is specifically favorable due to the fast access speed, high integration density, and zero standby power consumption. Recently, STT-RAM buffers for routers in Network-on-Chipp (NoC) systems and hybrid designs with SRAM and have been extensively employed to achieve mutually complementary features of various memory technologies, and control the efficiency of intra-router latency and system power consumption. With the implementation of Processing inMemory facilitated by STT-RAM, in this paper, we peculiarly drive the execution from processors to the STT RAM-based on-chip routers to enhance the application performance. The hybrid buffer designed in routers, we additionally exhibit system-level approaches, consisting of an ILP model and polynomial-time heuristic algorithms, to adjust the application mapping and scheduling on NoCs, to enhance system performance-energy efficiency. Network overhead induced by flit argument in typical communication frameworks can be ideally evaded by calculating the contended flits in intermediate routers; in the meantime, the pressure of heavy load on processors can be laid-back by assigning partial operations to routers, such that network latency and system power consumption can be drastically reduced. Experimental results show that application schedule length and system energy consumption can be decreased by $35: 62 \%, 32: 87 \%$ on average, respectively, in wide-ranging experiments on PARSEC standard applications. In particular, the realizations of application performance and energy efficiency, typically $36: 44 \%$ and $33: 19 \%$, for the CNN application AlexNet have substantiated the feasibility and effectiveness of our bestowed approaches [19].

Many core processor develops a conventional platform for cloud computing applications. However, the strategy of high performance and supportable inter-core communication networks is still a challenging issue. Optical Network on Chip $(\mathrm{ONoC})$ is an assuring chip-scale optical communication technology which is having high bandwidth competency and energy efficiency. In this paper, we proposed a Wavelength Reused Hierarchical ONoC architecture, WRH-ONoC. It controls the non-blocking wavelength-routed _-router and the hierarchical networking to reutilize the finite number of available wavelengths. In WRH-ONoC, all the cores are categorized into multiple subsystems, and the cores in the existing subsystem are directly interrelated by employing a single router for non-blocking communication. For inter-subsystem communication, all subsystems are additionally correlated through multiple _-routers and gateways hierarchically. Therefore, the available wavelengths can be used again in various _-routers. Furthermore, WRHm-ONoC, an efficient extension with multicast competence is also designed. Given the numbers of cores and available wavelengths, we develop the minimum hardware necessities, the predictable adjacent delay, and the maximum data rate. Conceptual study and simulation results specify that 
Sujata. S.B et al ., International Journal of Advanced Trends in Computer Science and Engineering, 9(3), May - June 2020,4117 - 4127

WRH-ONoC accomplishes noticeable development on communication performance and sustainability, e.g., 46:0\% of reduction on zero-load delay and 72:7\% of enhancement on throughput for 400 cores with satisfactory hardware cost and energy overhead [20].

Recently, Through-Silicon-Via (TSV) has been more popular to deliver faster inter-layer communication in three-dimensional Networks-on-Chip (3D NoCs). However, the area overhead of TSVs decreases wafer's employment and output which influences the design of 3D architectures utilizing a huge number of TSVs such as homogeneous 3D NoCs topologies. Similarly, more memory is required for 3D routers and thus they are more power-driven than traditional $2 \mathrm{D}$ routers. On the other hand, the area and performance benefits of $2 \mathrm{D}$ and $3 \mathrm{D}$ router architectures are combined in hybrid 3D NoCs that employs a finite number of TSVs. Present hybrid architectures experience higher packet delays as they do not reflect dynamic communication designs of various applications and their NoC resource utilization. We designed a new algorithm to analytically produce hybrid 3D $\mathrm{NoC}$ topologies for a given application such that the vertical connections are reduced while the NoC performance is not sacrificed. The proposed algorithm evaluates the target application and produces hybrid architectures by efficiently reallocating the vertical links and buffer areas based on their exploitations. Additionally, the algorithm has been computed with synthetic and different real-world traffic forms. Experimental results depict that the designed algorithm produces optimized architectures with lower energy consumption and a substantial decrease in packet delay in contrast to the existing solutions [21]. Three-dimensional networks on chip (3D-NoCs) have been designed as an extremely scalable solution to report communication issues in contemporary systems-on-chip. Through-silicon via (TSV) is typically implemented as a practical technology allowing vertical connection among NoC layers. However, TSV-based architectures normally show high exposure to transient and permanent faults produced by aging effects, thermal violations, manufacturing drawbacks, or even transient fault sources. Therefore, TSV-based architectures request for robust routing structures capable of satisfying operation under random failure patterns. In this paper, we present FL-RuNS, a fault-tolerant routing scheme for accomplishing $100 \%$ packet delivery under an unconfined set of runtime and permanent vertical link failures. The proposed scheme employs the notion of vertical link declaration to notify nodes in the network of the health condition of vertical links. This system is efficient and gradually reconfigures the complete network without any packet loss. FL-RuNS compels a smaller number of asymmetric virtual channels to realize both deadlock freedom and accessibility. Correspondingly, FL-RuNS presents one-flit-dedicated virtual channels, which are utilized as a gateway buffer in case of TSVs breakdowns. The experimental results have established that FL-RuNS exhibits better consistency in contrast to the recently designed fault-tolerant routing algorithm. Furthermore, the hardware synthesis is achieved by employing a commercial $28-\mathrm{nm}$ technology library that illustrates a reasonable power and area overhead concerning the non-fault-tolerant reference line [22].

Constant transistor scaling facilitates computer architecture to integrate a large number of cores on a chip. On-chip communication bandwidth requirement increases as the number of cores on a chip and application difficulty increases. The packet-switched network on chip (NoC) is visualized as a scalable and cost-effective communication structure for multi-core architectures with several hundred cores. In this chapter, we concentrate on an on-chip communication architecture strategy and initiate the reader to understand some important concepts of $\mathrm{NoC}$ architecture. This is monitored by a debate on the generally utilized power-saving techniques employed for NoCs and the disadvantages and constraints of these techniques. We then focus on performance expansion through the intellectual mapping of applications on multi-core architectures. We conclude the chapter with a conversation of various application explicit on-chip interconnect design approaches [23]. The incorporation of a large number of electronic components on a single chip has developed incomplete and complex systems on a single chip.

Since System-on-Chip ( $\mathrm{SoC}$ ) is usually battery-powered the Network-on-Chip (NoC) becomes one of the important tasks for energy proficiency and its communication subdivision. We demonstrate a review that gives a complete picture of the modern energy-efficient NoC architectures and techniques, such as the routing algorithms, buffered and bufferless router architectures, fault tolerance, switching approaches, voltage sources, and voltage-frequency scaling. The main aim of the study is to tutor the readers with the modern design-improvements that are employed to reduce the power consumption in the NoCs [24]. The proposal of efficient architectures for communication in on-chip multiprocessors system includes several tasks relating to the internal router functions which are employed in Network on Chip (NoC) set-up. The on-chip router is intended to provide per-flit processing with improved granularity. The quality of service undergone through the application level depends on the competences of the router to evade congestion and to guarantee efficient data-flow control. Therefore, an advanced router architecture is required to accomplish the requested QoS. This paper suggests an internal router architecture, for on-chip communication, employing a flow-control mechanism for preventing congestion by considering QoS. It portrays the internal functions of this router for best output flit scheduling and its ability to apply per-class service for inbound flows. The paper concentrates mainly on the description and performance study of two proposed structures for data flow control that can be employed with the designed router architecture. The results exhibited in this paper demonstrate that the application of these proposed techniques in $\mathrm{NoC}$ achieves a remarkable development in the measured 
adjacent QoS. We perform an extensive comparison of the proposed results with the existing schemes published in the work to illustrate that the proposed result improves on these, maintaining a remarkable tradeoff with the hardware characteristics when designed with $45 \mathrm{~nm}$ integration technology [25].

Deflection routing is an assuring methodology for energy and hardware efficient NoCs. Future VLSI designs will have an increased vulnerability to failures and breakdowns. The intrinsic redundancy of NoCs can be utilized to tolerate such failures. The non-fault-tolerant CHIPPER router architecture is extended to facilitate fault-tolerance. Based on deflection routing this architecture is designed and a permutation network is employed instead of a crossbar. In contrast to crossbar-based design, a permutation network permits a faster and smaller router design. Simulations of an $8 \times 8$ network and more than 30.000 flit injections depict, that our router architecture is feasible with existing crossbar-based fault-tolerant router architectures [26].

A standard Network-on-Chip (NoC) router employs input buffers to accumulate in-flight packets. These buffers increase the performance but use considerable power. It is feasible to ignore these buffers when they are empty, decreasing dynamic power, but static buffer power and dynamic power when buffers are used. To increase energy efficiency, bufferless deflection routing eliminates input buffers, and instead employ deflection (misrouting) to resolve conflict. However, at high network load, deflections produce redundant network hops, declining power and reducing performance. In this work, we propose a novel NoC router design referred to as a minimally-buffered deflection (MinBD) router. This router unifies deflection routing with a small "side buffer," which is much smaller than typical input buffers. A MinBD router set some network traffic that is then deflected in this side buffer, by considerably reducing deflections. Only a part of the traffic is buffered by the router, thus buffer area is resourcefully utilized than a router that holds every flit in its input buffers. We assess MinBD against input-buffered routers of different sizes that implement buffer bypassing, a bufferless router, and a hybrid design, and demonstrates that MinBD is more energy-efficient than all previous designs, and has performance that advances the standard input-buffered router with the area and power close to the bufferless router [27].

With the movement from computation centric approaches to communication-centric approaches in the Chip Multi-Processor (CMP) period, the interconnect structure is acquiring more importance. An efficient $\mathrm{NoC}$ in terms of power, area and average flit latency influences the overall functioning of a CMP. In the present work, MinBSD - a slightly buffered, single cycle, deflection router is proposed. It includes various operations (Injection, Ejection, Preemption, Re-injection) in a single module to control the traffic successfully and guarantees a smooth flow of flits via router pipeline. It achieves an overlapped implementation of independent operations. These features not only make MinBSD function in a single cycle but also to reduce the critical path latency ensuring faster interconnect network. Experimental results illustrate that MinBSD decreases the average flit latency on real workloads reduces perish area and power consumption in contrast to the existing advanced marginally buffered deflection routers [28]. In this paper, we propose a network-on-chip (NoC) design and are compared with conventional network design, featuring comparisons and distinction between the two. As a preliminary study, we review network congestion in bufferless NoCs. We demonstrate that congestion expresses itself differently in an NoC than in conventional networks. Network congestion decreases system output in congested workloads for smaller NoCs (16 and 64 nodes) and confines the scalability of larger bufferless NoCs (256 to 4096 nodes) even when traffic has an area (e.g., when an application's required data is mapped to its nearby core in the network). We propose a novel source throttling-based congestion control process with application-level mindfulness that decreases network congestion to enhance system performance. Our approach enhances system performance by up to $28 \%$ ( $15 \%$ on average in congested workloads) in smaller NoCs, accomplishes linear output scaling in NoCs up to 4096 cores (attaining similar performance scalability to an NoC with large buffers), and decreases power consumption by up to $20 \%$. Therefore, we illustrate an effective application of a network-level concept, congestion control, to a class of networks - bufferless on-chip networks - that has not been deliberated before by the networking community [29]. As Chip Multiprocessors (CMPs) can scale ten to hundreds of nodes, the interconnect becomes an important factor in cost, energy consumption, and performance. The energy efficiency of the fundamental communication framework plays an important role in the implementation of multi-core systems. Recent work recommends a cost-effective buffer-less deflection routing. An advanced version of the configurable network Creation Tool (CONNECT) is designed as a buffer-less router that is feeble and efficient. Based on the parallel port allocation process Modified CONNECT within the routers is proposed, which permits a maximum number of flits to be deflected in a practical direction. Modified CONNECT is evaluated by employing unvarying, transpose and inverse traffic workloads against CONNECT, BLESS and Cheap- Interconnect Partially Permuting Router (CHIPPER) on a 4x4 mesh network. Modified CONNECT saves 30\% area, in contrast, to CONNECT with lower performance. Modified CONNECT also hoards $24 \%$ in the area against BLESS by maintaining the same performance [30].

To serve the performance and scalability requirement of the rapid technical development towards exascale and large data processing with the performance of typical metal-based interconnects (wireline), alternative interconnects fabrics, such as inhomogeneous three-dimensional integrated network-on-chip (3D NoC) and hybrid wired-wireless network-on-chip (WiNoC), have initiated as a cost-effective 
Sujata. S.B et al ., International Journal of Advanced Trends in Computer Science and Engineering, 9(3), May - June 2020,4117 - 4127

resolution for developing system-on-chip (SoC) design. However, these interconnect trade-off enhanced performance for cost by limiting the number of area and power-driven 3D routers and wireless nodes. Furthermore, the non-uniform distributed traffic in a chip multiprocessor (CMP) requests an on-chip communication set-up that can evade congestion under high traffic circumstances while possessing negligible pipeline delay at low-load conditions. Finally, in this paper, we design a low-latency adaptive router with a low-complexity single-cycle avoiding process to improve the performance degradation due to time-consuming $2 \mathrm{D}$ routers in such developing hybrid NoCs. The recommended router transmits a flit by employing dimension-ordered routing (DoR) in the bypass data path at low-loads. When bypassing is not available and the output port requires intra-dimension, the packet is routed significantly to avoid congestion. The router also possesses a simplified virtual channel allocation (VA) technique that outputs a non-speculative low-latency pipeline. The proposed router is capable to balance the traffic in hybrid NoCs by integrating low-complexity evading technique with adaptive routing, to accomplish low-latency communication under different traffic loads. The simulation depicts that the proposed router can reduce the execution time of applications by an average of $16.9 \%$ in contrast to low-latency routers, such as SWIFT. By decreasing the latency between 2D routers (or wired nodes) and 3D routers (or wireless nodes), the proposed router can enhance the performance efficiency in terms of average packet delay by an average of $45 \%$ (or 50\%) in 3D NoCs (orWiNoCs) [31].

The trading structure of a point-to-point network describes in what way packets flow through each node and is the most important element in determining the performance of the network. In this paper, we design and assess a novel switching scheme which is referred to as hybrid switching. Virtual cut-through and wormhole switching are dynamically combined in Hybrid switching to provide higher feasible throughput than wormhole, which considerably decreases the buffer area required at intermediate nodes in contrast to virtual cut-through. This scheme is driven in contrast to virtual cut-through and wormhole switching through cycle-level simulations and then evaluated by employing the same approaches. To demonstrate the practicality of hybrid switching, and to provide a common platform for simulating and executing a variety of routing and switching schemes, we have proposed SPIDER, a communication adapter constructed around a convention ASIC known as the Programmable Routing Controller (PRC) [32]. Chip multiprocessors (CMPs) contains a low-latency interconnect fabric network-on-chip (NoC) to reduce processor stand time on instruction and data accesses that are overhauled by the last-level cache (LLC). Due to NoC-induced delays, the packet-switched mesh interrelated performance of many-core processors is reduced, lower network delays are not accessible by the present circuit-switched interconnects as the time taken to set up a circuit is not obscured. To describe this issue, this work presents CIMA - a hybrid circuit-switched and packet-switched mesh-based interconnection network that allows low LLC access delays at a small area cost. CIMA employs virtual cut-through (VCT) switching for short request packets and is advantageous to circuit switching for longer, delay-sensitive response packets. Attempts are made to set-up the circuit corresponding to the response packet when the request is being accomplished by the LLC, CIMA. A circuit has already been set, by the time the request packet is served and the response gets ready, and as a result, the response packet encounters a short delay in the network. A thorough evaluation aiming at 64-core CMP operating scale-out workloads discloses that CIMA enhances system performance by $21 \%$ over the advanced hybrid circuit-packet-switched network [33].

We design a packet-switched scheme for single-chip systems which scales a random number of processors like resources. The proposal, which we refer to as Network-on-Chip (NOC), consists of both the architecture and the design procedure. The NOC architecture is an $\mathrm{m} \times \mathrm{n}$ mesh of switches and resources are allocated on the slots designed by the switches. We employ a direct layout of the 2-D mesh of switches and resources providing physical- architectural level design integration. Every switch is linked to one resource and four neighboring switches, and every resource is linked to one switch. A resource consists of processor core, memory, an FPGA, a convention hardware block or any other intellectual property (IP) block, which fits into the available slot and implies with the interface of the NOC. The NOC architecture is an on-chip communication set-up including the physical layer, the data link layer and the network layer of the OSI protocol stack. We outline the concept of a region, which inhabits an area of several numbers of resources and switches. This model acknowledges the NOC to occupy large resources such as large memory banks, FPGA areas, or special purpose computation resources with high-performance multi-processors. The NOC design method comprises two stages. In the first stage, a concrete architecture is derived from the general NOC model. The concrete architecture describes the number of switches and outlines of the network, the type, and shape of regions and the quantity and type of resources. The second stage of concrete architecture is mapped with the application to form a concrete product [34]. In this paper, we design a new on-chip communication scheme by sharing the resources of a conventional packet-switched network-on-chip between a packet-switched and a circuit-switched sub-network. The previous work guides packets according to the conventional packet-switching mechanism, while the later forwards packets over circuits which are directly recognized between two non-adjacent nodes by avoiding the intermediate routers. To reach the destination, a packet can switch many times between the sub networks. The circuits are constructed by employing a low-latency and low-cost setup-network. The network resources are divided between the two sub-networks by utilizing Spatial-Division Multiplexing (SDM). The design objective is to enhance the power and performance 
metrics of Network-on-Chip (NoC) architectures and from the advantage of power and scalability of packet-switched NoCs and higher communication performance of circuit-switching. The evaluation results exhibit a substantial decrease in power and latency over a conventional packet-switched NoC [35].

\section{PROPOSED NOC SINGLE MICRO ARCHITECTURE FOR 8x8 NoC}

The effective communication for packets transmission within the NoC from any source to any destination nodes, the throughput and latency are the main parameters which will decides the successful delivering of packets, hence in this research work, proposes two different microarchitectures are proposed which are cost effective and high packet delivery ratio. The effective microarchitectures are buffer and bufferless by using $\mathrm{DB}^{2} \mathrm{R}$ NoC and costless direction findings. Each microarchitectures designs are shown in Figure1 and Figure 2.

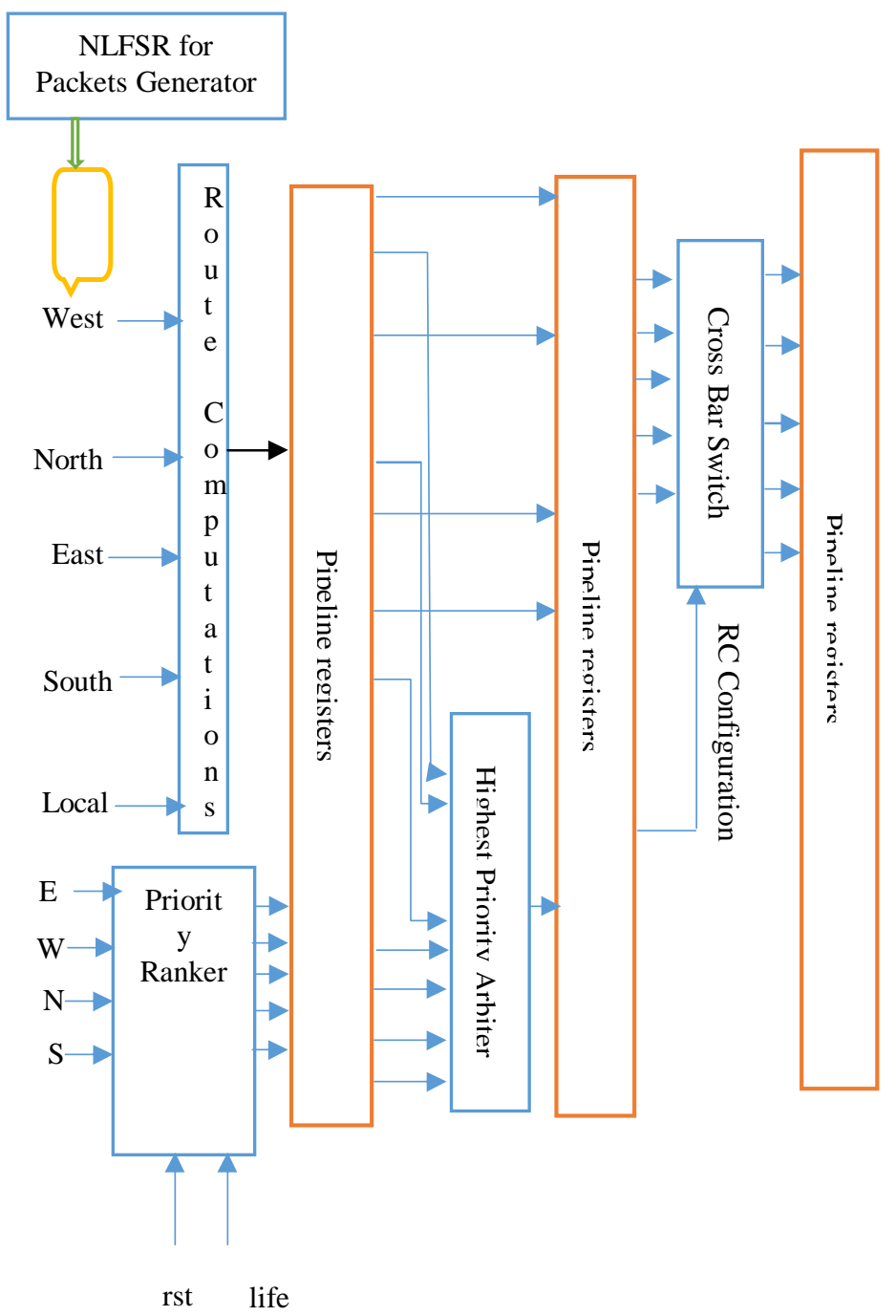

Figure1: Proposed Single Microarchitecture for $8 \times 8$ NoC by using Bufferless with priority allocation.
In bufferless, the routing operation performs in two stages such as flit ranking and selection of ports. For every cycle. The flit bit arriving to router is ranked and stored as highest priority and each flit performs the productivity at output port. Once the flits bits are ranked and output ports are available, then each flit will be assigned to each router based on highest ranking as depicts in Fig.2. The Fig.2. represents the microarchitecture for priority from highest to lowest, the highest priority arbiter for bufferless and it is second stage connected to next stage after produces and assign a output ports a priority in the first stage priority. The assignment of ports is based on highest to lowest order, the current output port is act as input at each arbiter cell based on matrix availability (matrxA) and the number of input port and effective port of output matrix (rmatrix) are shown in Figure 2.

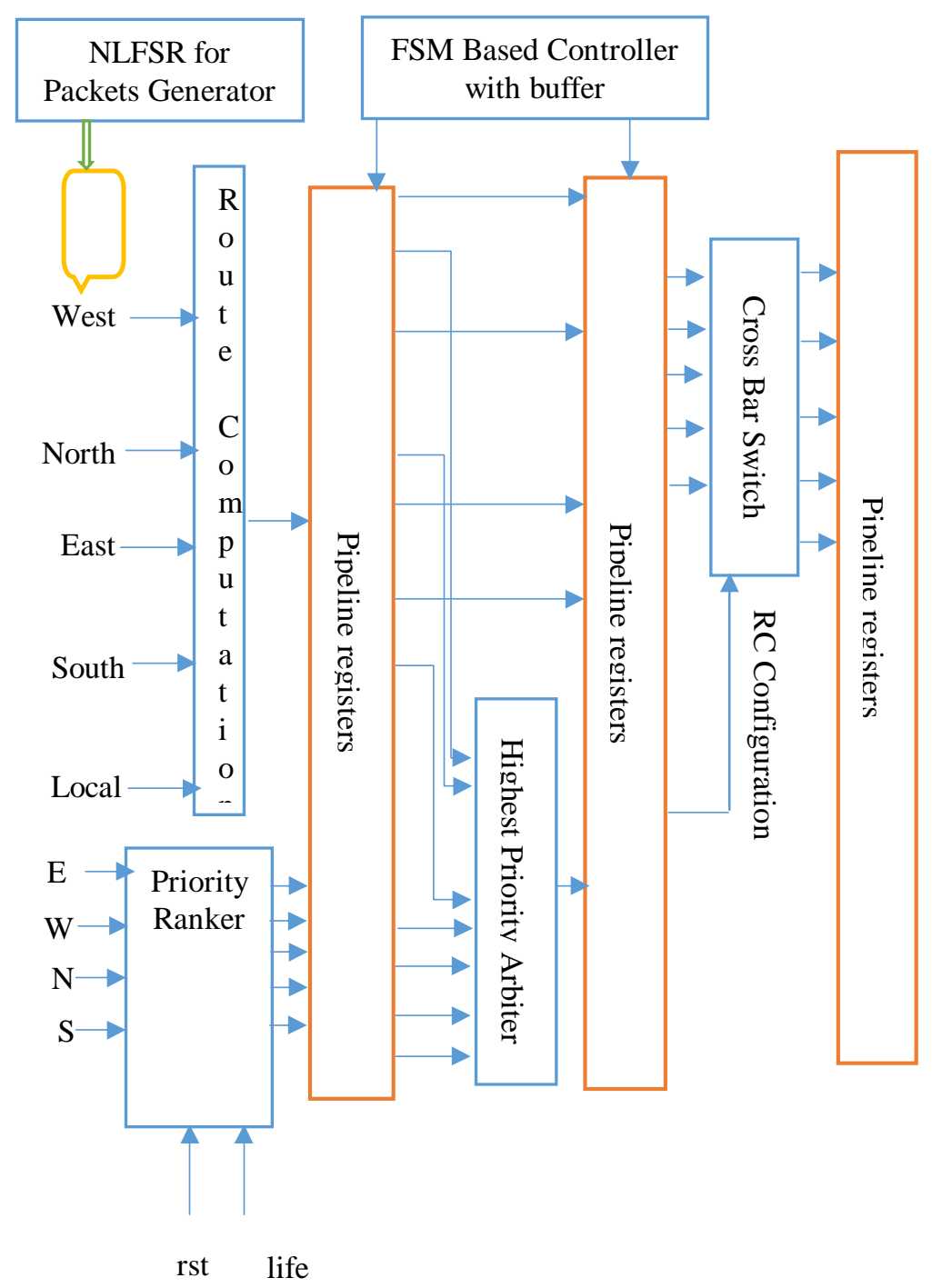

Figure 2: Proposed Single Microarchitecture for $8 \times 8$ NoC by using buffered with priority allocation.

The buffered NoC microarchitecture router performs main three stages of operations as follows.

First stage: When incoming flit and the flit in the buffer are valid as shown in the controller circuit shown in the Fig.2. 
Sujata. S.B et al ., International Journal of Advanced Trends in Computer Science and Engineering, 9(3), May - June 2020, 4117 - 4127

The control signal selects the flit which is already stored in the buffer for routing from source to destination. If "schedule_at_must" bit is set to high logic then control signal selects only "schedule_at_must" at highest priority and same will be updated in the priority table in the rank priority.

Second stage: The flits which are received and to be switched, priorities, output port list and "schedule_at_must" bit from first stage which whose are having highest priority, then the whole concept works as same as bufferless except "schedule_at_must" bit. So in this stage, this bit will set highest priority and routes to next node for transmission.

Third stage: This stage is different from bufferless in terms of crossbar design. In buffered, if "schedule_at_must" bit is set in the previous stage or control select signal force to set then "schedule_at_must" bit will set in the third stage therefore all flits will have priority in the priority ranker and every input and output ports can assign ports to avoid deadlock problems and congestions in the routing.

\section{CONCLUSION}

After successful survey on NoC's, it is found that different routing algorithms for packets transmissions form one router to another router, different switching techniques for direction finding and crossbar for mapping of input ports and output ports are detailed analyzed. It is also observed that there is slight changes in terms of architecture level but for routing and switching uses different algorithms like round robin, virtual cut through, warm whole, dynamically reconfigurable, flexible direction order and mesh. The deep survey on buffered and bufferless NoC's has better performance and it is easy to optimize the power consumption, delay and area. Bufferless NoC architectures are efficiency and high speed and is can be incorporated in multiprocessor system on chips and chip multiprocessor to perform complex operations. Finally, we would like to propose the modified $\mathrm{DB}^{2} \mathrm{R} \mathrm{NoC}$ and direction of finding for feasible, which can easily integrate into the conventional $\mathrm{NoC}$ desings. The proposed architecture are low cost, high speed, low delay and very less memory utilization.

\section{REFERNCES}

[1]. Thomas Moscibroda.at.al, "A Case for Bufferless Routing in On-Chip Networks", ISCA'09, June 20-24, 2009, Austin, Texas, USA.Copyright 2009 ACM 978-1-60558-526-0/09/06.

[2]. Juan Fang.at.al, "Hybrid Network-on-Chip: An Application-Aware Framework for Big Data", Hindawi, Complexity Volume 2018, Article ID 1040869, 11 pages, https://doi.org/10.1155/2018/1040869

[3]. Jing Lin.at.al, "Making-a-stop: A new bufferless routing algorithm for on-chip network", J. Parallel Distrib. Comput. 72 (2012) 515-524, 2012 Elsevier Inc. https://doi.org/10.1016/j.jpdc.2012.01.001
[4]. Rose George Kunthara.at.al, "ReDC: Reduced Deflection CHIPPER Router for Bufferless NoCs", 978-1-5386-6575-6/18, 2018 IEEE.

[5]. Alexander Shpiner.at.al, "On the Capacity of Bufferless Networks-on-Chip", IEEE TRANSACTIONS ON PARALLEL AND DISTRIBUTED SYSTEMS, VOL. 26, NO. 2, FEBRUARY 2015.

[6]. Yu-Hsiang Kao.at.al, "Design of A Bufferless Photonic Clos Network-on-Chip Architecture", IEEE TRANSACTIONS ON COMPUTERS, Digital Object Identifier 10.1109/TC.2012.250 0018-9340/12, 2012.

[7]. Chaochao Feng.at.al, "Addressing Transient and Permanent Faults in NoC With Efficient Fault-Tolerant Deflection Router", IEEE TRANSACTIONS ON VERY LARGE SCALE INTEGRATION (VLSI) SYSTEMS, Digital Object Identifier 10.1109/TVLSI.2012.2204909, 1063-8210, 2012 IEEE.

[8]. Dominic DiTomaso.at.al, "Resilient and Power-Efficient Multi-Function Channel Buffers in Network-on-Chip Architectures", IEEE TRANSACTIONS ON COMPUTERS, information: DOI 10.1109/TC.2015.2401013, IEEE Transactions on Computers, 0018-9340 , 2015 IEEE.

[9]. Anh T. Tran.at.al, "Achieving High-Performance On-Chip Networks With Shared-Buffer Routers", IEEE TRANSACTIONS ON VERY LARGE SCALE INTEGRATION (VLSI) SYSTEMS, 0.1109/TVLSI.2013.2268548, 1063-8210, 2013 IEEE.

[10].1063-8210/\$31.00 @ 2013 IEEE.at.al, "System-Level Buffer Allocation for Application-Specific Networks-on-Chip Router Design", IEEE TRANSACTIONS ON COMPUTER-AIDED DESIGN OF INTEGRATED CIRCUITS AND SYSTEMS, VOL. 25, NO. 12, DECEMBER 2006.

[11].Chih-Hao Chao.at.al, "Routing-Based Traffic Migration and Buffer Allocation Schemes for 3-D Network-on-Chip Systems With Thermal Limit”, IEEE TRANSACTIONS ON VERY LARGE SCALE INTEGRATION (VLSI) SYSTEMS, VOL. 21, NO. 11, NOVEMBER 2013. https://doi.org/10.1109/TVLSI.2012.2227852

[12].Mohammad Arjomand and Hamid Sarbazi-Azad, "Power-Performance Analysis of Networks-on-Chip with Arbitrary Buffer Allocation Schemes", IEEE TRANSACTIONS ON COMPUTER-AIDED DESIGN OF INTEGRATED CIRCUITS AND SYSTEMS, VOL. 29, NO. 10, OCTOBER 2010. https://doi.org/10.1109/TCAD.2010.2061171

[13].Cunlu Li.at.al, "RoB-Router : A Reorder Buffer Enabled Low Latency Network-on-Chip Router", IEEE TRANSACTIONS ON PARALLEL AND DISTRIBUTED SYSTEMS, 1045-9219, 2018 IEEE.

[14].Chun-Wei Wu.at.al, “ A Hybrid Multicast Routing Approach with Enhanced Methods for Mesh-Based Networks-on-Chip", IEEE TRANSACTIONS ON COMPUTERS, MANUSCRIPT

TC-2017-11-0610-R1, 0018-9340, 2018 IEEE. 
Sujata. S.B et al ., International Journal of Advanced Trends in Computer Science and Engineering, 9(3), May - June 2020, 4117 - 4127

[15].Davide Zoni.at.al, "CUTBUF: Buffer Management and Router Design for Traffic Mixing in VNET-based NoCs", 10.1109/TPDS.2015.2468716, IEEE Transactions on Parallel and Distributed Systems, 1045-9219, 2015 IEEE

[16].Yu-Yin Chen, at.al, "Path-Diversity-Aware Fault-Tolerant Routing Algorithm for NOC systems", IEEE TRANSACTIONS ON PARALLEL AND DISTRIBUTED SYSTEMS, 10.1109/TPDS.2016.2588482, 1045-9219, 2016 IEEE.

[17].Anastasios Psarras.at.al, "Networks-on-Chip With Double-Data-Rate Links",IEEE TRANSACTIONS ON CIRCUITS AND SYSTEMS-I: REGULAR PAPERS, 10.1109/TCSI.2017.2734689, 1549-8328, 2017 IEEE.

[18].Zhiliang Qian.at.al, "FSNoC: A Flit-Level Speedup Scheme for Networkon-Chips Using Self-Reconfigurable Bidirectional Channels", IEEE TRANSACTIONS ON VERY LARGE SCALE INTEGRATION (VLSI) SYSTEMS, 10.1109/TVLSI.2014.2351833, 1063-8210, 2014 IEEE.

[19].Lei Yang.at.al, "Optimal Application Mapping and Scheduling for Network-on-Chips with Computation in STT-RAM based Router", IEEE TRANSACTIONS ON COMPUTERS, 10.1109/TC.2018.2864749, 0018-9340, 2018 IEEE

[20].Feiyang Liu.at.al, "Wavelength-Reused Hierarchical Optical Network on Chip Architecture for Manycore Processors", DOI 10.1109/TSUSC.2017.2733551, IEEE Transactions on Sustainable Computing, 2377-3782, 2017 IEEE

[21].Michael Opoku Agyeman.at.al, "Performance and Energy Aware Inhomogeneous 3D Networks-on-Chip Architecture Generation", 10.1109/TPDS.2015. 2457444, IEEE Transactions on Parallel and Distributed Systems, 2015.

[22].Alexandre Coelho.at.al, "FL-RuNS: A High-Performance and Runtime Reconfigurable Fault-Tolerant Routing Scheme for Partially Connected Three-Dimensional Networks on Chip", IEEE TRANSACTIONS ON NANOTECHNOLOGY, 1536-125X, Volume 18, 2019.

https://doi.org/10.1109/TNANO.2019.2931271

[23]. Haseeb Bokhari and Sri Parameswaran, Network-on-Chip Design, Springer Science+Business Media Dordrecht 2017 S. Ha, J. Teich (eds.), Handbook of Hardware/Software Codesign, DOI 10.1007/978-94-017-7267-9_16.

[24]. Assad Abbas.at.al, "A survey on energy-efficient methodologies and architectures of network-on-chip", Computers and Electrical Engineering, ttp://dx.doi.org/10.1016/j.compeleceng.2014.07.012, 0045-7906/2014 Elsevier Ltd.

[25]. Ahmed Aldammas.at.al, "The efficiency of buffer and buffer-less data-flow control schemes for congestion avoidance in Networks on Chip", Journal of King Saud University Computer and Information Sciences, 2016, 28, 184-198 http://dx.doi.org/10.1016/j.jksuci.2015.11.002, 1319-1578,Production and hosting by Elsevier B.V.

[26]. Armin Runge, "FaFNoC: a Fault-tolerant and Bufferless Network-on-chip", Procedia Computer Science 56 ( 2015 ) $397-402,2015$.

[27].Chris Fallin.at.al, "MinBD: Minimally-Buffered Deflection Routing for Energy-Efficient Interconnect".

[28]. Gnaneswara Rao Jonna.at.al, "Minimally Buffered Single-Cycle Deflection Router", 978-3-9815370-2-4/DATE14/2014 EDAA.

[29]. George Nychis.at.al, On-Chip Networks from a Networking Perspective: Congestion and Scalability in Many-Core Interconnects, SIGCOMM'12, August 13-17, 2012, Helsinki, Finland. Copyright 2012 ACM 978-1-4503-1419-0/12/08

[30].Marwa Shaheen.at.al, "Modified CONNECT: New Bufferless Router for NoC-Based FPGAs", 978-1-5386-7392-8/18, 2018 IEEE

[31].Michael Opoku Agyeman.at.al, "Extending the Performance of Hybrid NoCs beyond the Limitations of Network Heterogeneity", Journal of Low Power Electronics and Applications, www.mdpi.com /journal/jlpea, Appl. 2017, 7, 8; doi:10.3390/jlpea7020008

[32].Kang G. Shin.at.al, "Analysis and Implementation of Hybrid Switching", IEEE TRANSACTIONS ON COMPUTERS, VOL. 45, NO. 6, JUNE 1996.

[33].Pejman Lotfi-Kamran.at.al, "An Efficient Hybrid-Switched Network-on-Chip for Chip Multiprocessors", Appears in IEEE Transactions on Computers, vol. 65, no. 5, pp. 1656-1662, May 2016 (DOI: 10.1109/TC.2015.2449846).

[34]. Shashi Kumar.at.al, "A Network on Chip Architecture and Design Methodology", Proceedings of the IEEE Computer Society Annual Symposium on VLSI (ISVLSI02), 0-7695-1486-3/02, 2002 IEEE.

[35]. Mehdi Modarressi.at.al, "A Hybrid Packet-Circuit Switched On-Chip Network Based on SDM", 978-3-9810801-5-5/DATE09, 2009 EDAA. 